\title{
Network meta-analysis and pharmacoeconomic evaluation of antibiotics for the treatment of patients infected with complicated skin and soft structure infection and hospital-acquired or ventilator-associated penumonia
}

Ying Zhang ${ }^{1 \dagger}$, Yan Wang ${ }^{1,2+}$, Mieke L. Van Driel ${ }^{3}$, Treasure M. McGuire ${ }^{2,4,5}$, Tao Zhang ${ }^{1}$, Yuzhu Dong ${ }^{1}$, Yang Liu', Leichao Liu', Ruifang $\mathrm{HaO}^{1}$, Lu Cao ${ }^{6}$, Jianfeng Xing ${ }^{6 *}$ and Yalin Dong ${ }^{1 *}$ (i)

\begin{abstract}
Background: Infections due to methicillin-resistant Staphylococcus aureus (MRSA) cause serious health risks and significant economic burdens and the preferred drugs are still controversial.
\end{abstract}

Methods: We performed a network meta-analysis (NMA) to compare the efficacy and safety of antibiotics used to treat inpatients with complicated skin and soft structure infections (CSSSI) or hospital-acquired or ventilatorassociated pneumonia (HAPNAP). We also developed a decision tree model to assess the cost-effectiveness of antibiotics.

Results: Forty-nine randomized controlled trials met the inclusion criteria (34 for cSSSI, 15 for HAPNAP) and compared the efficacy and safety of 16 antibiotics. For cSSSI, NMA indicated that for clinical cure, linezolid was superior than vancomycin (odds ratio (OR) 1.55, 95\% confidence interval (Cl) 1.19-2.02), while tedizolid (OR 1.39, Cl 0.70-2.76) was similar to vancomycin. In terms of safety, there were no significant differences between any two interventions on total adverse events. Based on drug and hospital costs in America, the incremental costeffectiveness ratios (ICERs) per life-year saved for linezolid and tedizolid compared with vancomycin were US\$2833 and US\$5523. For HAPNAP, there were no significant effects either for clinical cure or for safety endpoints between linezolid and vancomycin in NMA. ICERs per life-year saved for linezolid compared with vancomycin were US\$2185.

Conclusion: In these clinical trials, considering efficacy, safety, and cost-effectivenes, linezolid and tedizolid showed their superiority in MRSA cSSSl; while linezolid might be recommended to treat MRSA pneumonia. Although vancomycin was not cost-effective in pharmacoeconomic evaluation, it is still the first-line treatment for MRSA infection in the clinical practice. This study might provide new insights of therapeutic choices for patients with MRSA infections whilst awaiting the arrival of higher quality evidence.

\footnotetext{
*Correspondence: xajdxj@@mail.xjtu.edu.cn; dongyalin@mail.xjtu.edu.cn;

2576042020@qq.com

†Ying Zhang and Yan Wang contributed equally to this work.

${ }^{6}$ Department of Pharmacy, Xi'an Jiaotong University, Xi'an 710061, China

'Department of Pharmacy, The First Affiliated Hospital of Xi'an Jiaotong

University, Xi'an 710061, China

Full list of author information is available at the end of the article
}

(c) The Author(s). 2019 Open Access This article is distributed under the terms of the Creative Commons Attribution 4.0 International License (http://creativecommons.org/licenses/by/4.0/), which permits unrestricted use, distribution, and reproduction in any medium, provided you give appropriate credit to the original author(s) and the source, provide a link to the Creative Commons license, and indicate if changes were made. The Creative Commons Public Domain Dedication waiver (http://creativecommons.org/publicdomain/zero/1.0/) applies to the data made available in this article, unless otherwise stated. 


\section{Introduction}

Methicillin-resistant Staphylococcus aureus (MRSA) infections have posed a global threat since the end of the last century. MRSA infections cause increased mortality, cost burden and longer hospital stay [1]. Resistance tracking by SENTRY highlights the increasing prevalence of MRSA for two infections in which S. aureus predominates: complicated skin and soft-structure infections (cSSSIs); and hospital-acquired pneumonia (HAP) or ventilator-associated pneumonia (VAP) $[2,3]$.

The Infectious Diseases Society of America (IDSA) [4] published clinical practice guidelines for the treatment of MRSA infections, providing a practical basis for management. The glycopeptide vancomycin has been recommended by IDSA guidelines of MRSA treatment for many decades due to its excellent antibacterial activity [5]. However, in the latest decade, minimum inhibitory concentration (MIC) 'creep' among susceptible strains to vancomycin has been observed among MRSA isolates in USA, this has been consistently associated with increased mortality $[6,7]$.

In addition, there has recently been a sharp rise in the incidence of infections caused by MRSA $[8,9]$. This has resulted in increased use of vancomycin and the emergence of vancomycin resistant $S$. aureus $[9,10]$. As a consequence, there has been an emergence of new antibiotics to combat the evolving resistance of this challenging pathogen [11]. Linezolid, daptomycin, tigecycline, new glycopeptides such as telavancin and ceftaroline have been approved to treat MRSA infections. While meta-analyses [12-15] which have compared the efficacy and safety of vancomycin with linezolid or other antibiotics has been published; the results of these studies are not consistent and definitive conclusions cannot be drawn.

In this paper, we compared different antibiotics used for the treatment of MRSA infections using the technique of network meta-analysis (NMA) to determine which antibiotic(s) achieve the superior therapeutic effect. This approach can simultaneously assess multiple interventions in combination with direct and indirect evidence, thereby synthesizing available evidence. In addition, we developed a pharmacoeconomic analysis model from the patient perspective to investigate the cost-effectiveness of these antibiotics using clinical efficacy data generated by the NMA and medical data.

\section{Materials and methods}

\section{Search strategy and selection criteria}

A network meta-analysis of randomized controlled trials (RCTs) was performed in compliance with the Preferred Reporting Items for Systematic Reviews and Meta-analyses (PRISMA) guidelines [16]. We searched PubMed, EMBASE, and the Cochrane Central Register of Controlled Trials for RCTs published up to September 2018, using the search strategies described in Additional file 1: Appendix A. We also searched ClinicalTrials.gov for any relevant completed studies and hand searched the references from the retrieved RCTs.

\section{Study selection and data extraction}

Infections qualifying for this research were cSSSIs [17] and HAP/VAP in adult patients $[18,19]$. However, most of the studies we included were published prior to the Food and Drug Administration (FDA) guidance on designing RCTs to evaluate drugs for cSSSIs [20], with the results that the definition of cSSSI varied between studies. Therefore, we did not present a clear definition to avoid misunderstanding. HAP is defined as pneumonia that occurs $48 \mathrm{~h}$ or more after admission, which was not incubating at admission [21, 22]. VAP refers to pneumonia that occurs within $48-72 \mathrm{~h}$ after endotracheal intubation [21, 23]. To minimise clinical heterogeneity and because it is difficult to predict to what extent the increased prevalence of community-associated MRSA (CA-MRSA) will be accompanied by enhanced toxicity [24-27], we did not evaluate CA-MRSA infections.

The following eligibility criteria were applied: RCTs including adult patients with MRSA-related infections, and involving antibiotics with anti-MRSA activity. Studies were excluded if: (1) non-English language, (2) involving colonization or infection prevention, (3) pharmacokinetic/ pharmacodynamic studies, (4) the research only evaluated pharmacoeconomics or pooled analysis. Two researchers ( $\mathrm{YZ}$ and $\mathrm{YW}$ ) independently assessed the citations against the above eligibility criteria. Disagreements about study selection and data extraction were resolved through consensus.

The following data were extracted: authors, year of publication, patient population, study design, baseline characteristics, interventions (antibiotics for MRSA infections), clinical outcomes and sponsorship.

\section{Outcomes and quality assessment}

The primary outcome was clinical success at test of cure (TOC) in the modified intention-to-treat (mITT) population, which was determined cured and improved. Cured was defined as resolution of the clinical signs and symptoms of infection when compared with baseline; improved was defined as improvement in two or more, but not all, clinical signs and symptoms of infection when compared with baseline. The intent-to-treat (ITT) population included all patients randomized into the study. The mITT population was randomized patients receiving at least one dose of the study drug. The secondary endpoints included clinical efficacy in the clinically evaluable (CE) population and microbiological eradication in the microbiologically evaluable (ME) population. We also evaluated overall and serious adverse events and all-cause mortality in the safety population as safety 
outcomes. Since the definitions of mortality were different in each trial, we did not give specific time frame of mortality. The safety population was the primary population for all the safety analyses, and consisted of all patients who were dosed with study drug, irrespective of randomization. Since the included RCTs used different definitions for adverse events, such as nephrotoxicity and thrombocytopenia, the safety was assessed according to each RCT's own definition.

We evaluated the quality of included RCTs using the Cochrane risk of bias assessment tool in Review Manager version 5.3 .

\section{Pharmacoeconomic analysis model}

We conducted a decision-analytic model from the patient perspective to assess the outcomes of antibiotic therapy with antibiotics in patients with MRSA infections using TreeAge Pro 2011 (TreeAge Software, Inc., MA, USA). The decision tree model followed first-line and second-line therapy for MRSA cSSSI or HAP/VAP (see Additional file 1: Figure B.1).

Vancomycin was considered as comparator in the MRSA cSSSI model and the pneumonia model. The cure rate of the first-line treatment for the comparator was extracted from the pooled data of pairwise meta-analysis. The ORs of clinical cure for the comparator versus each antibiotic were obtained from NMA to generate the probabilities for comparator agents. The key outcomes of lifetime costs and life-years (LYs) saved for the two types of MRSA infections. The incremental cost-effectiveness ratios (ICERs) per LY saved was calculated, which was used to compare the performance of treatment strategies. We did not adjust for quality of life because we assumed that survivors of MRSA infections are unlikely to have long-term consequences related to this condition (i.e., utility value $=1$ ) [28]. We considered each treatment strategy resulting in an effect size less than the willingness-to-pay (WTP) threshold $(\$ 50,000)$ to be acceptable [29]. One-way sensitivity analyses were conducted to test how variation in one variable could affect model results. Probabilistic sensitivity analysis was carried out with 1000 times of Monte Carlo simulations to evaluate the impact of all variables simultaneously. The model and associated deterministic sensitivity analysis were developed from the patient perspective, with all costs are presented in 2017 US dollars with a conversion rate of 3\% [30] (additional details of the cost-effectiveness methodology in Additional file 1: Appendix B).

\section{Statistical analysis}

We conducted two types of meta-analyses. First, we performed pairwise meta-analysis with a random-effects model. The estimates of primary and secondary outcomes were determined using odds ratios (OR) and their corresponding 95\% confidence intervals (CI). Secondly, we conducted a random-effects network meta-analysis for direct and indirect comparisons [31]. In order to estimate the rank order for all interventions, surface under the cumulative ranking curve (SUCRA) probabilities were reported for primary outcome and safety outcome [32]. To check for the presence of inconsistency, we used the transitivity assumption to assess consistency of our assumption by comparing direct and indirect overall effects [33]. A common estimate (the tau [ $\tau]$ value) was assumed to evaluate the heterogeneity for all comparisons. To examine the robustness of the estimates with different baseline characteristics, sensitivity network meta-analyses for primary outcomes were performed on the following variables: sex ratio, age and restricting to low-risk of bias studies. We used Stata (Version 13.0) for the analyses (additional details of the statistical analysis of meta-analysis in Additional file 1: Appendix C).

\section{Results \\ Characteristics of included trials}

Of 3879 potentially relevant articles identified through the literature search, 3794 were excluded after the initial screening (Fig. 1). We reviewed the full text of the remaining 85 studies. We excluded 36 studies for the following reasons: pooled studies $(n=16)$, non-adult patients $(n=10)$, reviews or meta-analyses $(\mathrm{n}=10)$. Finally, 49 trials studying 16 different antibiotics met the criteria (34 for cSSSI, 15 for pneumonia) and were included in the NMA. The main characteristics of the included studies are shown in Additional file 1: Appendix D. The eligible comparison networks with MRSA cSSSI and pneumonia respectively are shown in Figs. 2 and 3.

The risk of bias summary and graph are listed in Additional file 1: Appendix E. Some studies were assessed as unclear risk of bias as they did not provide details about randomization, allocation concealment and blinding. The majority of studies are randomized, double-blind, controlled trials. Six studies reported evidence of blinding of participants and personnel. Most of the studies were sponsored by pharmaceutical companies (42 RCTs, 87\%).

\section{Pairwise and network meta-analysis of cSSSI}

For cSSSI, all 34 included studies reported clinical cure rate in the mITT population. Pairwise meta-analysis showed that linezolid was more effective than vancomycin (OR 1.23, 95\% CI 1.09-1.50, $I^{2}=0.0 \%$ ) (Table 1), which was consistent with the results of the NMA (OR 1.55, 95\% CI 1.19-2.02) (Fig. 4). Moreover, among patients with MRSA cSSSI, omadacycline was found to have a superior clinical response compared to all antibiotics except trimethoprim/sulfamethoxazole plus rifampicin (SXT/rifampin) (Fig. 4). Only 14 studies assessed the microbiological eradication of the ME population. 


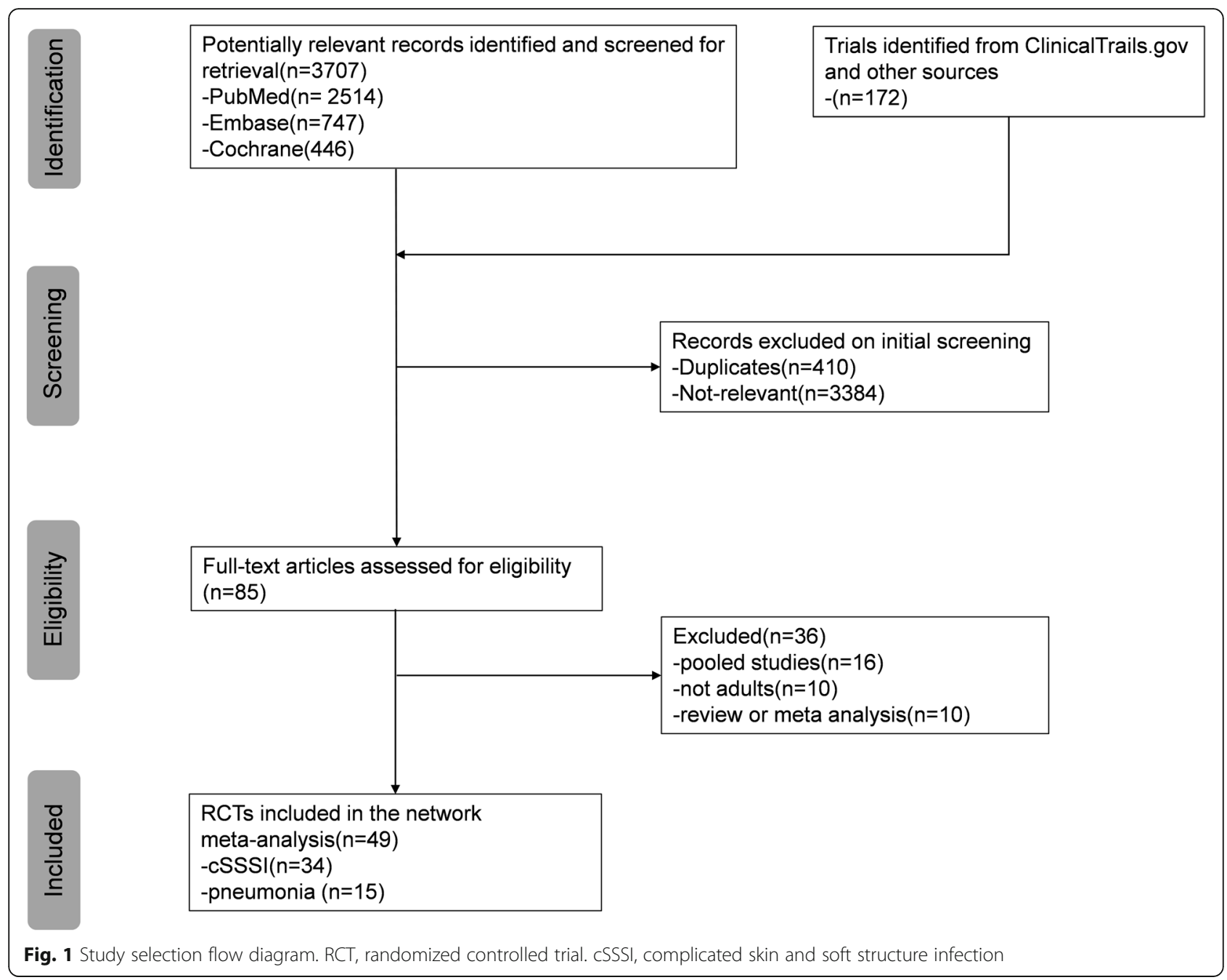

Pairwise meta-analysis showed that linezolid was significantly superior to vancomycin (OR 1.93, 95\% CI 1.26$2.96)$ in the microbiological response, which was consistent with the results of NMA (OR 2.42, 95\% CI 1.214.86) (see Additional file 1: Table F.1 and Figure F.1).

The majority of studies reported data on adverse events. There were no significant differences between any two interventions in terms of all-cause mortality rates in MRSA cSSSI patients. Telavancin had a higher total number of adverse events compared to vancomycin both in pairwise (OR 1.35, 95\% CI 1.14-1.60) and network meta-analyses (OR 1.25, 95\% CI 1.10-1.73) (see Additional file 1: Table F.2). The incidence of nephrotoxicity was similar between linezolid and vancomycin in head-to-head comparisons. Risk for thrombocytopenia was not statistically significantly different among linezolid and others in pairwise meta-analysis. Gastrointestinal adverse events were common with all included antibiotics in the treatment of MRSA cSSSI. (see Additional file 1: Table F.2).

The efficacy and safety of 15 interventions for the treatment of MRSA cSSSI are presented via the surface under the cumulative ranking curve (SUCRA) values which display the probability of each increasing rank order for efficacy and safety (see Additional file 1: Tables F.3 and F.4). In terms of efficacy, NMA found antibiotics ranked first to fourth via SUCRA values were omadacycline, SXT/ rifampin, JNJ-Q2, and oxazolidinone. In terms of safety, the lowest ranked antibiotic was telavancin.

\section{Pairwise and network meta-analysis of HAP/VAP}

In the smaller HAP network of studies, pairwise metaanalysis showed that for clinical cure rate linezolid was similar to vancomycin in the mITT population (OR 1.11, 95\% CI $0.91-1.34, I^{2}=0.0 \%$ ) (Table 1 ), which was consistent with the NMA results (OR 1.20, 95\% CI 0.961.50) (Fig. 5). In addition, the NMA of pneumonia trials found that for clinical cure rate there was no difference between teicoplanin and linezolid or teicoplanin and vancomycin (Fig. 5). The results of the head-to-head comparisons and NMA of different antibiotics in the ME population are listed in Additional file 1: Table G.1 and Figure G.1. Safety results of antibiotics are reported 


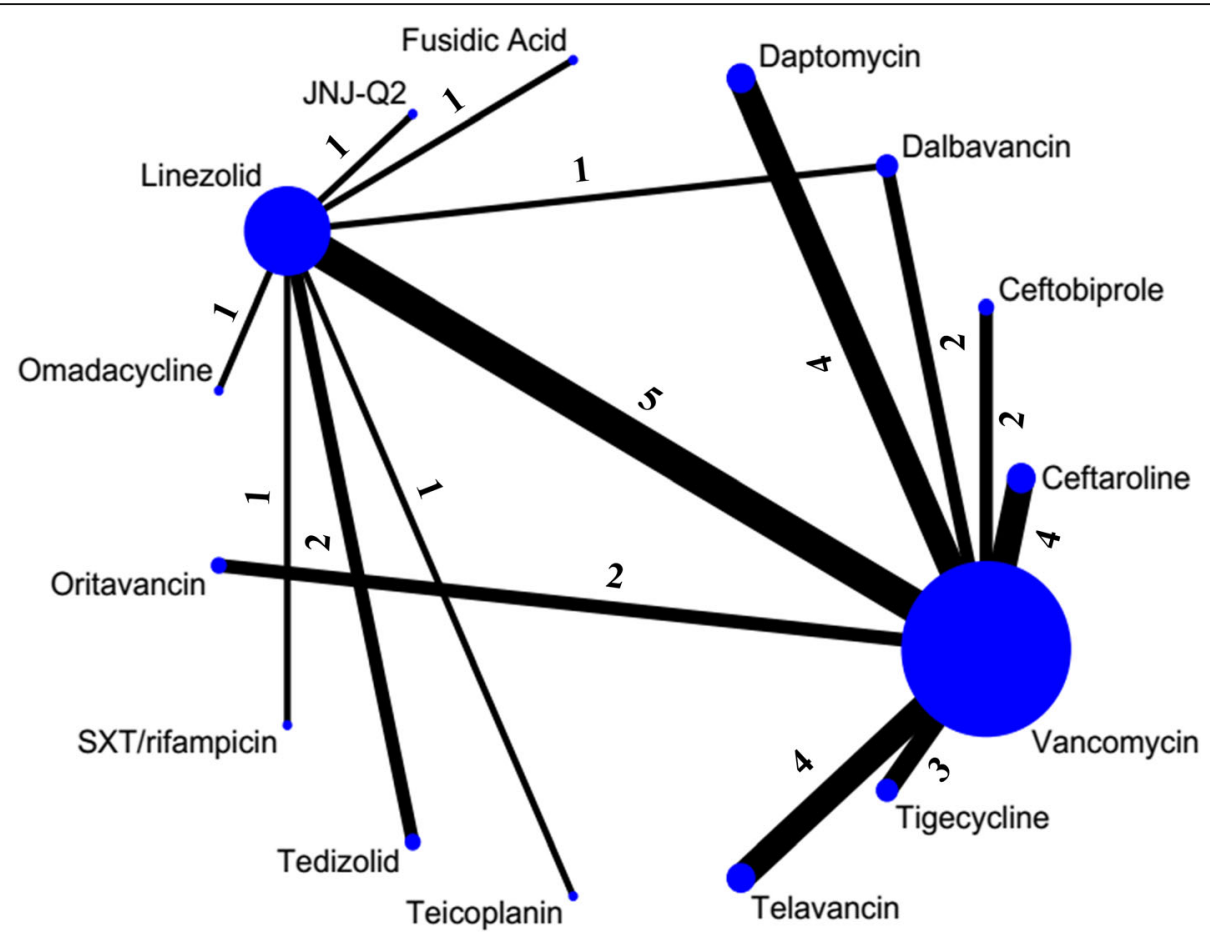

Fig. 2 Network plot of eligible comparisons for primary outcome in CSSSI group. The size of the node corresponds to the number of individual studies. The directly compared interventions are linked with a line, the width of which is proportional to the number of studies assessing respective comparisons. Numbers above the lines indicate studies. SXT, trimethoprim/sulfamethoxazole. JNJ-Q2, a novel fluoroquinolone

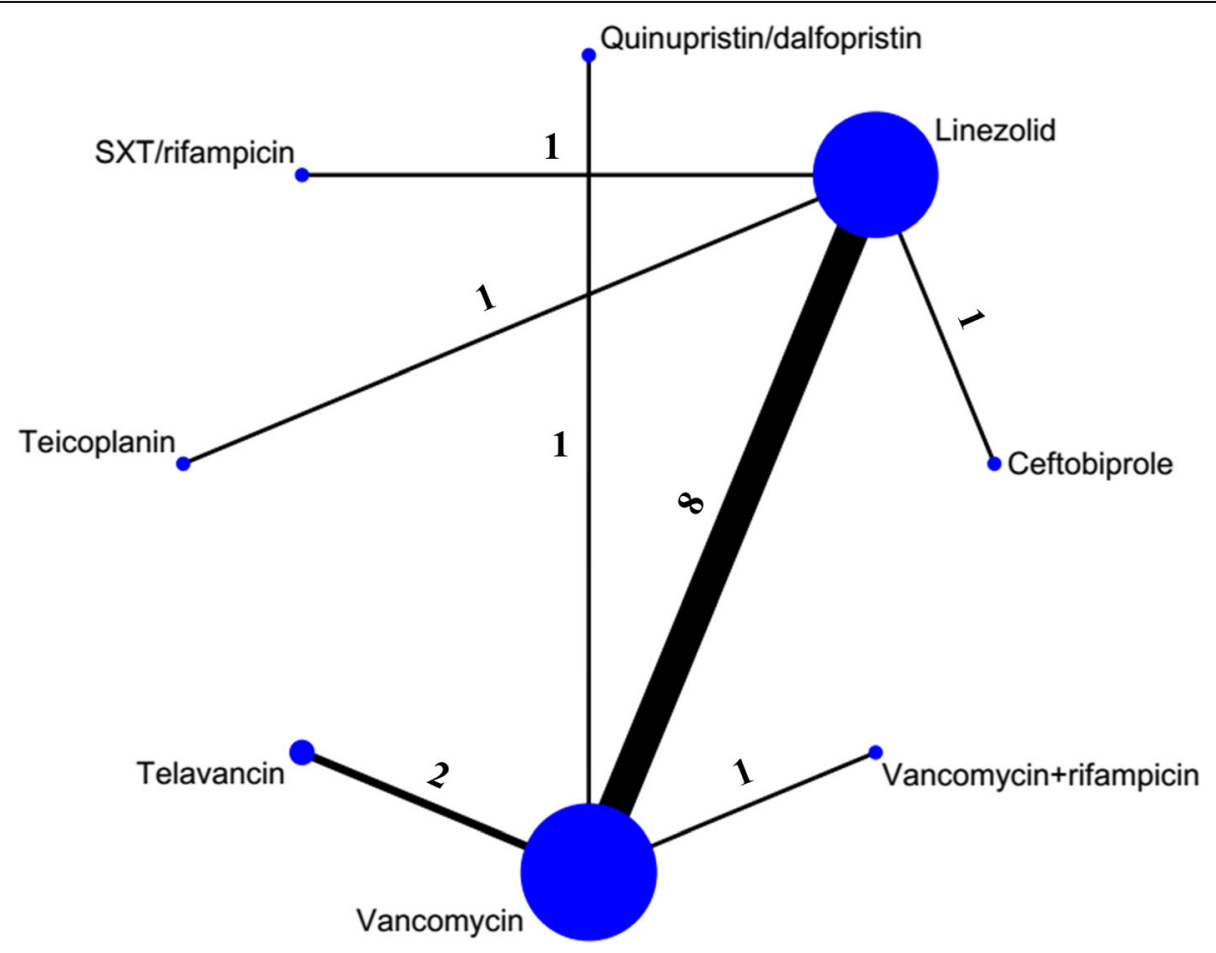

Fig. 3 Network plot of eligible comparisons for primary outcome in HAPNAP group. The size of the node corresponds to the number of individual studies. The directly compared interventions are linked with a line, the width of which is proportional to the number of studies assessing respective comparisons. Numbers above the lines indicate studies. SXT, trimethoprim/sulfamethoxazole 
Table 1 Pairwise meta-analysis of antibiotics of clinical cure in cSSSI and HAPNAP in mITT population

\begin{tabular}{|c|c|c|c|c|}
\hline Treatment comparisons & Number of studies & $\begin{array}{l}\text { Pairwise meta-analysis } \\
\text { Odds ratio }(95 \% \mathrm{Cl})\end{array}$ & $P$ value & $\begin{array}{l}\text { Heterogeneity } R^{2}, \% \text { (variation in } \mathrm{OR} \\
\text { attributable to heterogeneity) }\end{array}$ \\
\hline \multicolumn{5}{|l|}{ cSSSI } \\
\hline Vancomycin vs Oritavancin & 2 & $0.96(0.59,1.56)$ & 0.867 & 0.0 \\
\hline Vancomycin vs Daptomycin & 4 & $1.04(0.79,1.36)$ & 0.756 & 0.0 \\
\hline Vancomycin vs Telavancin & 4 & $0.86(0.61,1.24)$ & 0.430 & 0.0 \\
\hline Vancomycin vs Tigecycline & 3 & $1.15(0.79,1.66)$ & 0.451 & 0.0 \\
\hline Vancomycin vs Ceftaroline & 4 & $0.94(0.71,1.26)$ & 0.695 & 0.0 \\
\hline Vancomycin vs Dalbavancin & 2 & $1.00(0.77,1.31)$ & 0.973 & 0.0 \\
\hline Vancomycin vs Ceftobiprole & 2 & $0.78(0.33,1.82)$ & 0.565 & 0.0 \\
\hline Linezolid vs Vancomycin & 5 & $1.23(1.09,1.50)$ & 0.006 & 0.0 \\
\hline Linezolid vs Dalbavancin & 1 & $0.84(0.39,1.82)$ & 0.673 & - \\
\hline Linezolid vs SXT/rifampicin & 1 & $0.70(0.16,2.95)$ & 0.627 & - \\
\hline Linezolid vs Omadacycline & 1 & $0.36(0.03,4.18)$ & 0.417 & - \\
\hline Linezolid vs Fusidic Acid & 1 & $2.71(0.83,8.89)$ & 0.099 & - \\
\hline Linezolid vs JNJ-Q2 & 1 & $0.95(0.45,1.94)$ & 0.682 & - \\
\hline Linezolid vs Tedizolid & 2 & $1.09(0.58,2.02)$ & 0.780 & 0.0 \\
\hline Linezolid vs Teicoplanin & 1 & $0.52(0.15,1.79)$ & 0.234 & - \\
\hline \multicolumn{5}{|l|}{ HAPNAP } \\
\hline Vancomycin vs Telavancin & 2 & $1.02(0.85,1.23)$ & 0.822 & 0.0 \\
\hline Vancomycin vs Vancomycin/rifampicin & 1 & $1.49(0.72,3.06)$ & 0.278 & - \\
\hline Vancomycin vs Quinupristin/dalfopristin & 1 & $1.03(0.71,1.51)$ & 0.858 & - \\
\hline Linezolid vs Vancomycin & 8 & $1.11(0.91,1.34)$ & 0.301 & 0.0 \\
\hline Linezolid vs Ceftobiprole & 1 & $0.53(0.17,1.57)$ & 0.251 & - \\
\hline Linezolid vs Teicoplanin & 1 & $0.38(0.07,2.16)$ & 0.279 & - \\
\hline Linezolid vs SXT/rifampicin & 1 & $1.77(0.25,12.45)$ & 0.562 & - \\
\hline
\end{tabular}

CSSSI complicated skin and soft structure infection, HAPNAP hospital-acquired or ventilator-associated pneumonia, m/TT modified intention-to-treat population, JNJ-Q2 a novel fluoroquinolone, SXT trimethoprim/sulfamethoxazole

in Additional file 1: Table G.2. Most included studies reported on adverse events, and there was no significant difference between any two treatments for total adverse events. The incidence of nephrotoxicity of vancomycin was similar to linezolid (OR 1.02, 95\% CI 0.93-1.12). The difference was also not statistically significant between vancomycin and linezolid for the incidence of thrombocytopenia (OR 0.99, 95\% CI 0.86-1.55). Gastrointestinal adverse events were common with all antibiotics in the treatment of MRSA pneumonia (see Additional file 1: Table G.2).

NMA of MRSA pneumonia trials showed that ceftobiprole ranked first about efficacy, followed by teicoplanin, linezolid, and vancomycin (see Additional file 1: Table G.3). While vancomycin ranked first according to safety (see Additional file 1: Table G.4).

\section{Assessment of heterogeneity and inconsistency}

No significant heterogeneity within pairwise comparisons of antibiotics was found (Table 1). The values of heterogeneity variables for clinical cure rate in the mITT population, microbiological response in the ME population, clinical cure rate in the $\mathrm{CE}$ population and the incidence of total adverse events were $\tau=0.09, \quad \tau=0.33 \quad \tau=0.43, \quad \tau=0.18$, respectively (all values indicated moderate heterogeneity). In the inconsistency test only one closed loop (Dalbavancin-Linezolid-Vancomycin) was detected and the results are shown in Additional file 1: Appendix H. There were no substantial differences between direct and indirect treatment effect estimates and therefore no evidence of inconsistency in the cSSSI network. In the MRSA pneumonia network, indirect evidence was hardly obtained in the presence of direct evidence for any interventions, hence an inconsistency test was not performed.

\section{Sensitivity analysis}

The results of the post hoc sensitivity analysis of MRSA cSSSI and pneumonia are presented in 


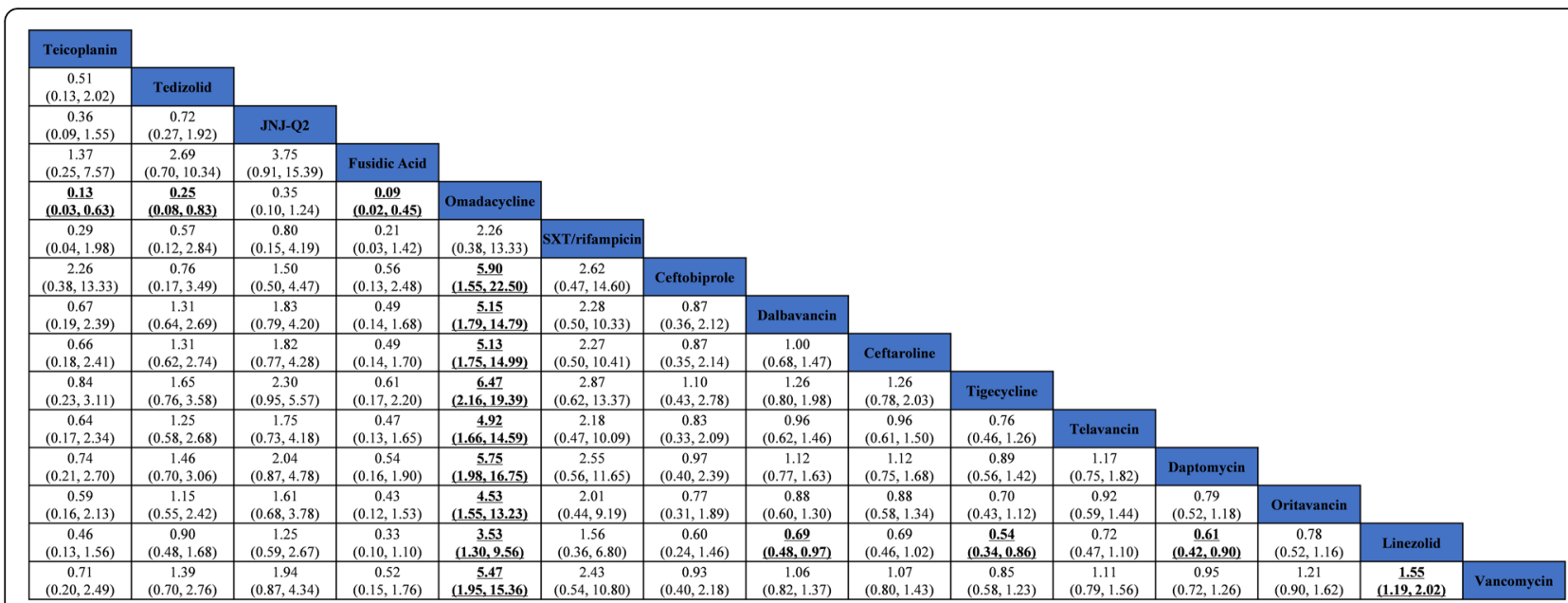

Fig. 4 Network meta-analysis of clinical cure in CSSSI in mITT population. Comparisons should be read from left to right. The safety estimate is located at the intersection of the column-defining treatment and the row-defining treatment. Significant results are in bold and underlined. cSSSI, complicated skin and soft structure infection. mITT, modified intention-to-treat population. JNJ-Q2, a novel fluoroquinolone. VAN, vancomycin. LIN, linezolid. CEF ${ }^{1}$, ceftaroline. CEF ${ }^{2}$, ceftobiprole. SXT/RIT, trimethoprim/sulfamethoxazole plus rifampicin. ORI, oritavancin. DAL, dalbavancin. DAP, daptomycin. TEL, telavancin. TIG, tigecyline. TEl, teicoplanin. OMA, omadacycline. FA, fusidic acid. JNJ-Q2, a novel fluoroquinolone. TED, tedizolid. $\mathrm{OR}$, odds ratio. $\mathrm{Cl}$, confidence interval

Additional file 1: Appendix F and G. In cSSSI, linezolid and tedizolid showed more effective than other antibiotics when high risk studies were excluded (i.e., SXT/rifampin in cSSSI). Linezolid and vancomycin were superior to other antibiotics in pneumonia after including the research with high-risk of bias (high risk studies were excluded, i.e., teicoplanin in pneumonia). The efficacy rankings were robust after adjusting for the mean age and sex ratios. The comparison-adjusted funnel plots showed no asymmetry in Additional file 1: Appendix I.

\section{Cost analysis}

\section{Base-case analysis}

Ten antibiotics (vancomycin, linezolid, tedizolid, telavancin, dalbavancin, oritavancin, ceftaroline, tigecycline, daptomycin, SXT/rifampin) were included in the MRSA cSSSI model. Exclusions were fusidic acid and teicoplanin as the NMA showed they were less effective; JNJ-Q2 was not marketed; although omadacycline was approved by FDA, it was not marketed currently; and ceftobiprole has been discontinued. The costs and clinical outcomes of the 10 treatments are summarized in Additional file 1:

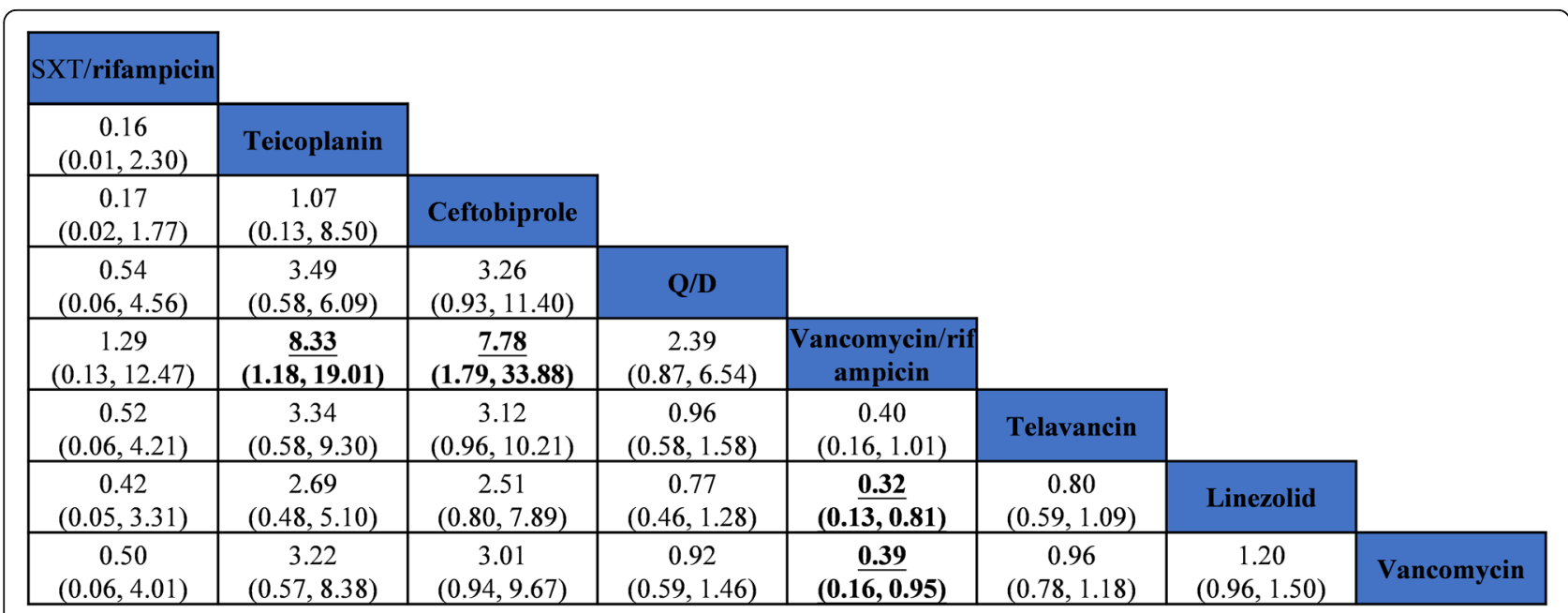

Fig. 5 Network meta-analysis of clinical cure in pneumonia in mITT population. Comparisons should be read from left to right. The safety estimate is located at the intersection of the column-defining treatment and the row-defining treatment. Significant results are in bold and underlined. mlTt, modified intention-to-treat population. VAN, vancomycin. LIN, linezolid. CEF², ceftobiprole. SXT/RIT, trimethoprim/sulfamethoxazole plus rifampicin. TEL, telavancin. TEl, teicoplanin. VAN/RIF, vancomycin plus rifampicin. Q/D, quinupristin/dalfopristin OR, odds ratio. Cl, confidence interval 
Table 2 Cost-effectiveness of antibiotics for treatment of cSSSI patients

\begin{tabular}{|c|c|c|c|c|c|}
\hline Treatment strategy & Total cost (USD) & Incremental cost (USD) & Total LY & LY saved $^{\mathrm{a}}$ & ICER per LY saved \\
\hline Vancomycin & 15,254 & & 7.52 & & \\
\hline SXT/rifampicin & 13,419 & -1836 & 7.68 & 0.16 & Dominant \\
\hline Linezolid & 16,387 & 1133 & 7.92 & 0.40 & 2833 \\
\hline Tedizolid & 17,353 & 2099 & 7.90 & 0.38 & 5523 \\
\hline Daptomycin & 17,238 & 1984 & 7.84 & 0.32 & 6200 \\
\hline Ceftaroline & 17,971 & 2717 & 7.82 & 0.30 & 9057 \\
\hline
\end{tabular}

CSSSI complicated skin and soft structure infection, USD US dollar, LY life-years, ICER incremental cost-effectiveness ratio, SXT trimethoprim/sulfamethoxazole

${ }^{a}$ Calculated as the average cost per patient and the average number of LY per patient in this strategy minus those of the treatment of vancomycin

Table B.1. Vancomycin was used as the baseline to calculate the incremental cost-effectiveness ratio (ICER) for other strategies, in accordance with current clinical guidelines [4]. Tigecycline, oritavancin, dalbavancin, telavancin, ceftaroline were less effective than vancomycin, and the ICERs per LY saved corresponded to $>$ US $\$ 50,000$. Therefore, these five options were not considered cost-effective treatment strategies. In the present analysis, SXT/rifampicin was the dominant strategy, however, there was insufficient data to recommend it since the RCT studying SXT/rifampicin was of high risk. The ICERs per LY saved for linezolid, tedizolid, daptomycin and ceftaroline relative to vancomycin corresponded to US\$2833, US\$5523, US\$6200 and US\$9057, respectively (Table 2). These five antimicrobial agents might be recommended for the treatment of MRSA cSSSI within a range of WTP thresholds.

For MRSA pneumonia, teicoplanin, vancomycin, linezolid, telavancin, SXT/rifampicin and quinupristin/ dalfopristin (Q/D) were evaluated in the cost analysis. The total costs and effectiveness of the five treatment options are shown in Additional file 1: Table S2. The ICERs were calculated relative to vancomycin, which was considered the baseline for other strategies. All antibiotics including telavancin, Q/D and SXT/rifampicin were subordinate to vancomycin except linezolid and teicoplanin, since the ICERs per LY saved corresponded to $>$ US $\$ 50,000$. In the present analysis, teicoplanin was the dominant strategy, however, we were unable to recommend this treatment due to the limited data with high risk study. The ICER per LY saved for using linezolid over vancomycin was US\$2185 (Table 3). These two antibiotics might be recommended for treatment of MRSA pneumonia.

\section{Sensitivity analysis}

Deterministic sensitivity analyses revealed that the results were most sensitive to the costs of antibiotics included in the economic analysis and the duration of treating MRSA cSSSI and HAP/VAP. For MRSA cSSSI, the unit cost of daptomycin more than US\$305 would result in more cost-effective compared with linezolid. In HAP/VAP, the unit cost of telavancin less than US\$320 would make it more cost-effective than linezolid. Other variations in a single model parameter had no substantial impact on the primary analyses both in cSSSI and pneumonia.

In cSSSI, probabilistic sensitivity analyses (PSA) displayed that SXT/rifampin, linezolid, tedizolid, daptomycin, ceftaroline, tigecycline, dalbavancin, oritavancin and telavancin had probabilities of 88.7, 87.7, 87.2, 86.7, 84.1, 72.4, $69.0,52.1$, and $50.0 \%$, respectively, of being cost-effective relative to vancomycin under the WTP threshold $(\$ 50,000)$. As for pneumonia, teicoplanin, linezolid, Q/D, telavancin and SXT/rifampin had probabilities of 83.2 , $60.0,34.3,26.3,17.5 \%$, respectively within the threshold.

\section{Discussion}

Our systematic review and NMA comprehensively evaluated the efficacy, safety, and cost-effectiveness of antibiotics used to treat MRSA cSSSI and pneumonia. We found that for clinical cure linezolid and tedizolid were superior to the other antibiotics in patients with cSSSI. In terms of safety, there was no significant difference between any two interventions for total adverse events. Our cost analysis showed that oxazolidinone and vancomycin were cost-effective in the treatment of MRSA infections and within a range of WTP thresholds. For MRSA pneumonia, the efficacy of linezolid and vancomycin were better than other antibiotics. As for safety,

Table 3 Cost-effectiveness of antibiotics for treatment of pneumonia patients

\begin{tabular}{llllll}
\hline Treatment strategy & Total cost (USD) & Incremental cost (USD) & Total LY & LY saved $^{\text {a }}$ & ICER per LY saved \\
\hline Vancomycin & 16,346 & & 9.84 & & Dominant \\
Teicoplanin & 12,487 & -3858 & 10.80 & 0.96 & 1.32 \\
Linezolid & 19,230 & 2884 & 11.16 & 2185 \\
\hline
\end{tabular}

USD US dollar, $L Y$ life-years, ICER incremental cost-effectiveness ratio

${ }^{a}$ Calculated as the average cost per patient and the average number of LY per patient in this strategy minus those of the treatment of vancomycin 
no significant difference was found between any of the treatments for total adverse events. In the cost-analysis, linezolid and vancomycin were cost-effective within a range of WTP thresholds.

cSSSIs are common infections in everyday clinical practice, resulting in high morbidity and health care cost [34]. We found that linezolid had better efficacy in patients with cSSSI, which is consistent with guideline recommendations. Our findings are consistent with a previous study [35] which highlighted that SXT/rifampicin and linezolid had similar efficacy but lower cost [36]. Nevertheless only one RCT included in our NMA directly compared SXT/rifampicin and linezolid, there is insufficient evidence to make any conclusion. A recent large meta-analysis [37] showed that linezolid was associated with a significantly higher clinical cure rate and reduced length of hospital stay compared with vancomycin for cSSSI. The improved skin penetration of linezolid and its high bioavailability may explain the improved clinical outcomes among patients receiving linezolid [38]. Similarly, linezolid may be a cost-effective alternative to vancomycin in the treatment of patients with cSSSI [39, 40], which was consistent with our analysis. Tedizolid, a novel oxazolidinone, showed comparable efficacy to linezolid in a phase 3 RCTs [41], which was consistent with our results. Omadacycline which belongs to a new class of compounds, aminomethyl-cyclines, is considered a promising drug for the treatment of severe MRSA infections [42]. RCTs found that omadacycline was more effective than linezolid; however, due to the limited number of studies in our NMA, we are unable to conclude whether omadacycline can be used as an alternative to linezolid. Although there was no significant difference between any of the treatments for total adverse events, attention should still be paid to some adverse events for antibiotics (e.g., thrombocytopenia for linezolid, nephrotoxicity for vancomycin).

Pneumonia is the second most common hospital-acquired infections in adults, contributing to inpatient mortality [43]. Previous studies compared the efficacy of linezolid and vancomycin in the treatment of MRSA pneumonia [44-47] with contradictory results. Using NMA we showed that the clinical response with linezolid is similar to vancomycin, which is consistent with guideline recommendations. Furthermore, our analysis showed that the efficacy of teicoplanin was better than the other interventions, but differences were not statistically significant. However, as only one RCT on teicoplanin was included, this remains to be further verified. Although there was no statistically significant difference among included antibiotics, we should pay attention to several adverse events for antimicrobial agents. Telavancin was approved by FDA for the treatment of hospital-acquired and ventilator-associated bacterial pneumonia caused by Staphylococcus aureus [48]. To our knowledge, telavancin has not been compared with linezolid in any clinical or observational studies. Our study compared these two drugs indirectly and found no significant differences in efficacy. Serious adverse events of telavancin can cause poor clinical outcomes among patients, which may limit its application in clinical practice. HAP caused an increase of $\$ 11,897$ to $\$ 25,072$ per incident and was the leading cause of death in all nosocomial infections [49]. In four pharmacoeconomic analyses comparing vancomycin and linezolid in the treatment of pneumonia, efficacy and cost data were obtained from published RCTs [50-53]. Our results confirmed the results of previous analyses and showed that despite its higher cost, linezolid was cost-effective for treatment of MRSA pneumonia.

Given the difficulties of assessing multiple RCTs to directly compare individual antibiotics used to treat severe MRSA infections, the use of NMA to compare the relative efficacy and safety of new and traditional drugs through indirect methods is recommended. Previous studies have evaluated the effectiveness and safety of antibiotics used for treating hospitalized adults with cSSSI or pneumonia $[14,15,54]$. However, timely evaluation of the latest treatment strategies in the era of antimicrobial resistance is crucial for treatment decision making.

Our study has several limitations. First, most of the research were published prior to the 2010 FDA guideline for designing RCTs to evaluate antibiotics for CSSSI [20]. As a consequence, the definition of cSSSI varied between studies. Second, the low quality of some research due to unclear bias risk and the possibility of publication bias, may jeopardize the validity of our conclusions. Findings from our analysis, however, were not affected by the results from the research with high-risk of bias via sensitivity analysis. Third, the confidence intervals for some antibiotics are really wide because of limited sample size. Therefore, we recommend that larger research should be included in order to make a more accurate conclusion. Fourth, due to various adverse events of involved antibiotics, we only describe the probability and costs associated with primary adverse events (e.g thrombocytopenia for linezolid or nephrotoxicity for vancomycin). Ignoring differences in the probability and costs of adverse events may influence results. Finally, our cost analysis only evaluated linezolid intravenous formulations. Linezolid has the main advantage of oral administration, with almost $100 \%$ bioavailability [55]. Given the high bioavailability of linezolid [56], future studies should consider the cost-effectiveness of oral dosage forms to comprehensively evaluate the clinical value of antibiotics for the treatment of MRSA infections. This study might provide new insights of therapeutic choices for patients with MRSA infections whilst awaiting the arrival of higher quality evidence. 


\section{Conclusions}

Considering efficacy, safety, and cost-effectivenes, linezolid and tedizolid showed their superiority in MRSA cSSSI; while linezolid might be recommended to treat MRSA pneumonia. Although vancomycin was not cost-effective in pharmacoeconomic evaluation, it is still the first-line treatment for MRSA infection in the clinical practice. The approach used in this study might assist in revisiting therapeutic choices for patients with MRSA infections while awaiting the arrival of higher quality evidence.

\section{Additional file}

Additional file 1: Supplementary material of network meta-analysis and pharmacoeconomic evaluation. (DOCX 2848 kb)

\section{Abbreviations}

ABSSSI: Acute bacterial skin and skin structure infections; $C A-$ MRSA: Community-associated MRSA; CE: Clinically evaluable; Cl: Confidence interval; cSSSI: Complicated skin and soft structure infections; FDA: Food and Drug Administration; HAP: Hospital-acquired pneumonia; ICER: Incremental cost-effectiveness ratio; IDSA: The Infectious Diseases Society of America; ITT: Intention-to-treat; LYs: Life years; ME: Microbiologically evaluable; MIC: Minimum inhibitory concentration; mITT: Modified intention-to-treat; MRSA: Methicillin-resistant Staphylococcus aureus; NMA: Network metaanalysis; OR: Odds ratio; PRISMA: Preferred reporting items for systematic reviews and meta-analyses; PSA: Probabilistic sensitivity analysis; Q/ D: Quinupristin/dalfopristin; RCT: Randomized controlled trial; SUCRA: Surface under the cumulative ranking curve; SXT: Trimethoprim/sulfamethoxazole TOC: Test of cure; WTP: Willingness-to-pay

\section{Acknowledgments}

Not applicable.

\section{Funding}

This study was supported by the National Natural Science Foundation of China [No. 81473177] and 2016 clinical research special award of Xi'an Jiaotong University, China (No. XJTU 1AF-CRF-2016 T-03).

\section{Availability of data and materials}

Data sharing is not applicable to this article as no datasets were generated or analysed during the current study.

\section{Authors' contributions}

YalinD was involved in the study conception and study design. YZ was involved in the study conception, study design, selection of trials, data collection, data analysis, initial draft of manuscript writing and critical revision of the manuscript. YW was involved in the study design, data collection, data analysis and initial draft of manuscript writing. TZ was involved in the study conception, data analysis, critical revision of the manuscript. YuzhuD was involved in the study conception, interpretation of the data and critical revision of the manuscript. YL was involved in the study conception and critical revision of the manuscript. LL was involved in the study conception and critical revision of the manuscript. RH, MLVD and TMM were involved in the critical revision of the manuscript. LC was involved in the critical revision of the manuscript. All authors read and approved the final manuscript.

\section{Ethics approval and consent to participate}

Not applicable.

\section{Consent for publication}

Not applicable.

\section{Competing interests}

The authors declare that they have no competing interests.

\section{Publisher's Note}

Springer Nature remains neutral with regard to jurisdictional claims in published maps and institutional affiliations.

\section{Author details}

${ }^{1}$ Department of Pharmacy, The First Affiliated Hospital of Xi'an Jiaotong University, Xi'an 710061, China. ${ }^{2}$ School of Pharmacy, University of Queensland, Brisbane, Queensland, Australia. ${ }^{3}$ Primary Care Clinical Unit, Faculty of Medicine, University of Queensland, Brisbane, Queensland, Australia. ${ }^{4}$ Faculty of Health Sciences \& Medicine, Bond University, Gold Coast, Queensland, Australia. ${ }^{5}$ Mater Pharmacy Services, Mater Health Services, Brisbane, Queensland, Australia. ${ }^{6}$ Department of Pharmacy, Xi'an Jiaotong University, Xi'an 710061, China.

Received: 14 February 2019 Accepted: 4 April 2019

Published online: 06 May 2019

\section{References}

1. Crum NF, Lee RU, Thornton SA, Stine OC, Wallace MR, Barrozo C, et al. Fifteen-year study of the changing epidemiology of methicillin-resistant Staphylococcus aureus. Am J Med. 2006;119:943-51.

2. Jones RN. Microbial etiologies of hospital-acquired bacterial pneumonia and ventilator-associated bacterial pneumonia. Clin Infect Dis. 2010;51(Suppl.1: S81-7.

3. Moet GJ, Jones RN, Biedenbach DJ, Stilwell MG, Fritsche TR. Contemporary causes of skin and soft tissue infections in North America, Latin America, and Europe: report from the SENTRY antimicrobial surveillance program (1998-2004). Diagn Microbiol Infect Dis. 2007;57:7-13.

4. Liu C, Bayer A, Cosgrove SE, Daum RS, Fridkin SK, Gorwitz RJ, et al. Clinical practice guidelines by the infectious diseases society of america for the treatment of methicillin-resistant Staphylococcus aureus infections in adults and children: executive summary. Clin Infect Dis. 2011;52:285-92.

5. Gould IM, David MZ, Esposito S, Garau J, Lina G, Mazzei T, et al. New insights into meticillin-resistant Staphylococcus aureus (MRSA) pathogenesis, treatment and resistance. Int J Antimicrob Agents. 2012;39: 96-104.

6. Steinkraus $G$, White $R$, Friedrich L. Vancomycin MIC creep in nonvancomycin-intermediate Staphylococcus aureus (VISA), vancomycinsusceptible clinical methicillin-resistant S. aureus (MRSA) blood isolates from 2001-05. J Antimicrob Chemother. 2007;60:788-94.

7. Kalil AC, Van Schooneveld TC, Fey PD, Rupp ME. Association between vancomycin minimum inhibitory concentration and mortality among patients with Staphylococcus aureus bloodstream infections: a systematic review and meta-analysis. JAMA. 2014;312:1552-64.

8. Chambers HF. The changing epidemiology of Staphylococcus aureus? Emerg Infect Dis. 2001;7:178-82.

9. Daum RS. Clinical practice. Skin and soft-tissue infections caused by methicillin-resistant Staphylococcus aureus. N Engl J Med. 2007:357:380-90.

10. Cosgrove SE, Carroll KC, Perl TM. Staphylococcus aureus with reduced susceptibility to vancomycin. Clin Infect Dis. 2004;39:539-45.

11. Tsoulas C, Nathwani D. Review of meta-analyses of vancomycin compared with new treatments for gram-positive skin and soft-tissue infections: are we any clearer? Int J Antimicrob Agents. 2015:46:1-7.

12. Agarwal R, Bartsch SM, Kelly BJ, Prewitt M, Liu Y, Chen Y, et al. Newer glycopeptide antibiotics for treatment of complicated skin and soft tissue infections: a systematic review, network meta-analysis and cost analysis. Clin Microbiol Infect. 2018;24:361-8.

13. An MM, Shen $H$, Zhang JD, Xu GT, Jiang YY. Linezolid versus vancomycin for meticillin-resistant Staphylococcus aureus infection: a meta-analysis of randomised controlled trials. Int J Antimicrob Agents. 2013;41:426-33.

14. Bally M, Dendukuri N, Sinclair A, Ahern SP, Poisson M, Brophy J. A network meta-analysis of antibiotics for treatment of hospitalised patients with suspected or proven meticillin-resistant Staphylococcus aureus infection. Int J Antimicrob Agents. 2012:40:479-95.

15. Falagas ME, Siempos II, Vardakas KZ. Linezolid versus glycopeptide or $\beta$ lactam for treatment of gram-positive bacterial infections: meta-analysis of randomised controlled trials. Lancet Infect Dis. 2008;8:53-66.

16. Hutton B, Salanti G, Caldwell DM, Chaimani A, Schmid CH, Cameron C, et al. The PRISMA extension statement for reporting of systematic reviews incorporating network meta-analyses of health care interventions: checklist and explanations. Ann Intern Med. 2015;162:777-84. 
17. US Department of Health and Human Services, Food and Drug Administration, Center for Drug Evaluation and Research. Guidance for industry. Acute bacterial skin and skin structure infections-developing drugs for treatment. 2010.

18. American Thoracic Society (ATS). Infectious Diseases Society of America (IDSA) guidelines for the management of adults with hospital-acquired, ventilator- associated and healthcare-associated pneumonia. Am J Respir Crit Care Med. 2005;171:388-416.

19. Masterton RG, Galloway A, French G, Street M, Armstrong J, Brown E, et al. Guidelines for the management of hospital-acquired pneumonia in the UK report of the working party on hospital-acquired pneumonia of the British Society for Antimicrobial Chemotherapy. J Antimicrob Chemother. 2008:62:5-34

20. US Food and Drug Administration CfDEaR. Guidance for industry. Acute bacterial skin and skin-structure infections: developing drugs for treatment 2010

21. Tablan OC, Anderson L, Besser R, Bridges C, Hajjeh R, CDC; Healthcare Infection Control Practices Advisory Committee. Guidelines for preventing health-care--associated pneumonia, 2003: recommendations of CDC and the healthcare infection control practices advisory committee. MMWR Recomm Rep. 2004;53:1-36.

22. Niederman MS. Guidelines for the management of respiratory infection: why do we need them, how should they be developed, and can they be useful? Curr Opin Pulm Med. 1996;2:161-5.

23. Craven DE, Kunches LM, Kilinsky V, Lichtenberg DA, Make BJ, McCabe WR. Risk factors for pneumonia and fatality in patients receiving continuous mechanical ventilation. Am Rev Respir Dis. 1986;133:792-6.

24. David MZ, Daum RS. Community-associated methicillin-resistant Staphylococcus aureus: epidemiology and clinical consequences of an emerging epidemic. Clin Microbiol Rev. 2010;23:616-87.

25. Otter JA, French GL. Molecular epidemiology of community-associated meticillinresistant Staphylococcus aureus in Europe. Lancet Infect Dis. 2010;10:227-39.

26. Klevens RM, Morrison MA, Nadle J, Petit S, Gershman K, Ray S, et al. Invasive methicillin-resistant Staphylococcus aureus infections in the United States. JAMA. 2007;298:1763-71

27. DeLeo FR, Otto M, Kreiswirth BN, Chambers HF. Community-associated meticillin-resistant Staphylococcus aureus. Lancet. 2010;375:1557-68.

28. Ingenix. National Fee Analyzer. Eden Prairie: Ingenix; 2011.

29. Shillcutt SD, Walker DG, Goodman CA, Mills AJ. Cost-effectiveness in lowand middle-income countries. Pharmacoeconomics. 2010;27:903-17.

30. Gold JES MR, Russell LB, Weinstein MC. Cost-effectiveness in health and medcine. New York: Oxford University Press; 1996

31. Caldwell DM, Ades AE, Higgins JP. Simultaneous comparison of multiple treatments: combining direct and indirect evidence. BMJ. 2005;331:897-900.

32. Chaimani A, Higgins JP, Mavridis D, Spyridonos P, Salanti G. Graphical tools for network meta-analysis in STATA. PLoS One. 2013;8:e76654

33. Turner RM, Davey J, Clarke MJ, Thompson SG, Higgins JP. Predicting the extent of heterogeneity in meta-analysis, using empirical data from the Cochrane database of systematic reviews. Int J Epidemiol. 2012;41:818-27.

34. Lee BY, Singh A, David MZ, Bartsch SM, Slayton RB, Huang SS, et al. The economic burden of community-associated methicillin-resistant Staphylococcus aureus (CA-MRSA). Clin Microbiol Infect. 2013;19:528-36.

35. Harbarth S, von Dach E, Pagani L, Macedo-Vinas M, Huttner B, Olearo F, et al. Randomized non-inferiority trial to compare trimethoprim/ sulfamethoxazole plus rifampicin versus linezolid for the treatment of MRSA infection. J Antimicrob Chemother. 2015;70:264-72.

36. von Dach E, Morel CM, Murthy A, Pagani L, Macedo-Vinas M, Olearo F, et al. Comparing the cost-effectiveness of linezolid to trimethoprim/ sulfamethoxazole plus rifampicin for the treatment of methicillin-resistant Staphylococcus aureus infection: a healthcare system perspective. Clin Microbiol Infect. 2017;23:659-66.

37. Yue J, Dong BR, Yang M, Chen X, Wu T, Liu GJ. Linezolid versus vancomycin for skin and soft tissue infections. Cochrane Database Syst Rev. 2016;(1)CD008056.

38. MacGowan AP. Pharmacokinetic and pharmacodynamic profile of linezolid in healthy volunteers and patients with gram-positive infections. J Antimicrob Chemother. 2003;51:ii17-25.

39. Wan Y, Li Q, Chen Y, Haider S, Liu S, Gao X. Economic evaluation among Chinese patients with nosocomial pneumonia caused by methicillinresistant Staphylococcus aureus and treated with linezolid or vancomycin: a secondary, post-hoc analysis based on a phase 4 clinical trial study. J Med Econ. 2016;19:53-62.

40. Bounthavong M, Zargarzadeh A, Hsu DI, Vanness DJ. Cost-effectiveness analysis of linezolid, daptomycin, and vancomycin in methicillin-resistant
Staphylococcus aureus: complicated skin and skin structure infection using Bayesian methods for evidence synthesis. Value Health. 2011;14:631-9.

41. Prokocimer P, De Anda C, Fang E, Mehra P, Das A. Tedizolid phosphate vs linezolid for treatment of acute bacterial skin and skin structure infections: the ESTABLISH-1 randomized trial. JAMA. 2013;309:559-69.

42. Villano S, Steenbergen J, Loh E. Omadacycline: development of a novel aminomethylcycline antibiotic for treating drug-resistant bacterial infections. Future Microbiol. 2016;11:1421-34.

43. Torres A, Ferrer M, Badia JR. Treatment guidelines and outcomes of hospital-acquired and ventilator-associated pneumonia. Clin Infect Dis. 2010; 51:S48-53.

44. Wunderink RG, Niederman MS, Kollef MH, Shorr AF, Kunkel MJ, Baruch A, et al. Linezolid in methicillin-resistant Staphylococcus aureus nosocomial pneumonia: a randomized, controlled study. Clin Infect Dis. 2012;54:621-9.

45. Falagas ME, Siempos II, Vardakas KZ. Linezolid versus glycopeptide or betalactam for treatment of gram-positive bacterial infections: meta-analysis of randomised controlled trials. Lancet Infect Dis. 2008;8:53-66.

46. Walkey AJ, O'Donnell MR, Wiener RS. Linezolid vs glycopeptide antibiotics for the treatment of suspected methicillin-resistant Staphylococcus aureus nosocomial pneumonia: a meta-analysis of randomized controlled trials. Chest. 2011;139:1148-55

47. Wang $Y$, Zou Y, Xie J, Wang $T$, Zheng $X, \mathrm{He} \mathrm{H}$, et al. Linezolid versus vancomycin for the treatment of suspected methicillin-resistant Staphylococcus aureus nosocomial pneumonia: a systematic review employing meta-analysis. Eur J Clin Pharmacol. 2015;71:107-15.

48. Wenzler E, Rodvold KA. Telavancin: the long and winding road from discovery to food and drug administration approvals and future directions. Clin Infect Dis. 2015;61:S38-47.

49. Klevens RM, Edwards JR, Richards CL Jr, Horan TC, Gaynes RP, Pollock DA, et al. Estimating health care-associated infections and deaths in U.S. hospitals, 2002. Public Health Rep. 2007;122:160-6.

50. Patel DA, Shorr AF, Chastre J, Niederman M, Simor A, Stephens JM, et al. Modeling the economic impact of linezolid versus vancomycin in confirmed nosocomial pneumonia caused by methicillin-resistant Staphylococcus aureus. Crit Care. 2014;18:R157.

51. Niederman MS, Chastre J, Solem CT, Wan Y, Gao X, Myers DE, et al. Health economic evaluation of patients treated for nosocomial pneumonia caused by methicillin-resistant Staphylococcus aureus: secondary analysis of a multicenter randomized clinical trial of vancomycin and linezolid. Clin Ther. 2014;36:1233-43.

52. Lyles A. Cost-effectiveness analysis of linezolid compared with vancomycin for the treatment of nosocomial pneumonia caused by methicillin-resistant Staphylococcus aureus. Clin Ther. 2007;29:384

53. Collins CD, Schwemm AK. Linezolid versus vancomycin in the empiric treatment of nosocomial pneumonia: a cost-utility analysis incorporating results from the ZEPHyR trial. Value Health. 2015;18:614-21.

54. Thom H, Thompson JC, Scott DA, Halfpenny N, Sulham K, Corey GR. Comparative efficacy of antibiotics for the treatment of acute bacterial skin and skin structure infections (ABSSSI): a systematic review and network meta-analysis. Curr Med Res Opin. 2015;31:1539-51.

55. Plouffe JF. Emerging therapies for serious gram-positive bacterial infections: a focus on linezolid. Clin Infect Dis. 2000;31:S144-9.

56. Mendes RE, Deshpande LM, Jones RN. Linezolid update: stable in vitro activity following more than a decade of clinical use and summary of associated resistance mechanisms. Drug Resist Updat. 2014:17:1-12.

\section{Ready to submit your research? Choose BMC and benefit from:}

- fast, convenient online submission

- thorough peer review by experienced researchers in your field

- rapid publication on acceptance

- support for research data, including large and complex data types

- gold Open Access which fosters wider collaboration and increased citations

- maximum visibility for your research: over $100 \mathrm{M}$ website views per year

At BMC, research is always in progress.

Learn more biomedcentral.com/submissions 